\title{
Characterization of ODS Steels after Gamma Irradiation for Application in ALLEGRO Reactor
}

\author{
V. Slugeñ*, I. Bartošová And J. Dekan \\ Institute of Nuclear and Physical Engineering, Faculty of Electrical Engineering and Information Technology, \\ Ilkovičova 3, 81219 Bratislava, Slovakia

\begin{abstract}
Oxide-dispersion strengthened steels characterization using various spectroscopic techniques is presented. Microstructure of $15 \%$ chromium oxide-dispersion strengthened steels was studied in term of vacancy defects presence and their accumulation after defined irradiation treatment, respectively. Studied materials originated from Kyoto University and studied via IAEA collaborative project focused on generation IV reactors (ALLEGRO). Samples were characterized "as received" by positron annihilation lifetime spectroscopy, the Mössbauer spectroscopy and their microstructure was examined by transmission electron microscopy as well. Samples were afterwards irradiated in Washington State University Nuclear Radiation Center via a strong gamma source (6 TBq). Damage induced by gamma irradiation was evaluated by positron lifetime measurements in emphasis on defect accumulation in the materials. We have demonstrated strong defect production induced by gamma irradiation which results from positron measurement data.
\end{abstract}

DOI: 10.12693/APhysPolA.131.1090

PACS/topics: 29.30.-h, 76.80.+y, 81.05.-t, 81.05.Bx, 82.80.Ej

\section{Introduction}

Oxide-dispersion strengthened steels (ODS) have been developed mainly for high temperature applications for advanced fusion reactor blanket components as well as the next generation fission reactor fuel cladding or gas cooled fast reactor prototype ALLEGRO materials [1].

The microstructure of these steels is enhanced by dispersed particles of $\mathrm{Y}_{2} \mathrm{O}_{3}$ which influence dislocation movement and also serve as trapping sites for positrons due to high positron affinity of yttrium. Therefore investigation by means of positron annihilation lifetime spectroscopy (PALS) provides a wide range of information. Positron measurements are supported by transmission electron microscopy (TEM) which provides visual evaluation and overall parameters of the samples and the Mössbauer spectroscopy (MS) analyses focused on materials microstructure.

The main aim of this study was the proper application of selected techniques for the microstructural characterisation of progressive materials.

\section{Experimental details}

Studied materials designated KOC originated from Kyoto University and were studied via IAEA collaborative project. Heat treatment involved hot extrusion at $1150^{\circ} \mathrm{C}$ and subsequent air cooling.

For TEM further sample preparation was needed. Thin steel foil with thickness of $0.1 \mathrm{~mm}$ was prepared by mechanical grinding. Afterwards targets with $3 \mathrm{~mm}$ diameter were further thickened in Tenupol 5 device in a solution of $300 \mathrm{ml} \mathrm{HNO}_{3}+700 \mathrm{ml} \mathrm{CH} 3 \mathrm{OH}$, voltage $15 \mathrm{~V}$ and electrolyte temperature $0{ }^{\circ} \mathrm{C}$. After cleansing in ethanol

*corresponding author; e-mail: vladimir.slugen@stuba.sk and drying, the sample was prepared for TEM. Investigations by TEM were carried out on JEOL 200CX electron microscope with accelerating voltage $200 \mathrm{keV}$. Selected area electron diffraction (SAED) for identification of lattice parameters supplemented TEM analyses.

The microstructure of all samples is very similar. The mean size of ferritic grains for samples KOC4-3, KOC5-3 and KOC6-3 is $d_{\text {str }}=550 \pm 25 \mathrm{~nm}, d_{\text {str }}=800 \pm 100 \mathrm{~nm}$ and $d_{s t r}=650 \pm 40 \mathrm{~nm}$, respectively. We also observed small globular particles, which show strong heterogeneity whether in size or distribution. Particle size varied from 2 to $300 \mathrm{~nm}$ however in all samples more than $60 \%$ of particles were $20-40 \mathrm{~nm}$ in diameter. SAED identified these particles to be $\mathrm{Y}_{2} \mathrm{O}_{3}$. Interestingly in sample 5-3 the $\mathrm{Y}_{2} \mathrm{O}_{3}$ phase was found in two modifications bcc and also fcc. Also a presence of quasi globular particles was acknowledged in samples KOC4-3 and KOC53 which precipitate mainly on ferritic grain boundaries. Due to chemical composition and SAED results we believe the secondary phase is that of $\mathrm{M}_{23} \mathrm{C}_{6}$. Analyses also provided some evidence of dislocation-precipitation interactions and interactions with $\mathrm{Y}_{2} \mathrm{O}_{3}$ particles.

\section{Results and discussion}

While ODS steels are complex systems with various elements as an additive, from the Mössbauer spectroscopy point of view, the iron bearing compounds of the steels, which are in detection limits of the method $(>1 \%)$, can be reduced to similar system as in dilute $\mathrm{Fe}-\mathrm{Cr}$ binary alloy.

Dilute iron alloys $\mathrm{Fe}-\mathrm{X}$, where $\mathrm{X}$ is a $3 d$ transition element, are intensively studied for last 40 years using the Mössbauer spectrometry [2]. Hyperfine magnetic fields $\left(B_{h f}\right)$ and isomer shifts (IS) at ${ }^{57} \mathrm{Fe}$ nuclei, are used for investigation of the changes in phase composition caused by $\mathrm{X}$ element in alloy structure by various authors [3-6].

Changes in parameters of the Mössbauer spectra in dilute alloys are assigned mainly to local interactions of 
atoms. Flinn and Ruby [7] noted that satellite lines in spectra of the $\mathrm{Fe}-\mathrm{Al}$ system could be described as the superposition of number of sextets for Fe atoms with a different number of $\mathrm{Al}$ atoms in the nearest neighborhood, where number of sextets is defined by binomial distribution. Later, this method was extended to other Fe-based alloys.

The samples for the Mössbauer experiments were prepared by scraping into powder form and were measured at room temperature using constant-acceleration Wissel Mössbauer spectrometer with the ${ }^{57} \mathrm{Co}(\mathrm{Rh})$ source in transmission geometry. The diameters of particles were less than $15 \mu \mathrm{m}$. All MS parameters are given in Table I. The spectra contain sextet of narrow lines, which practically does not differ from spectrum of pure bcc Fe. Besides that, broad, asymmetrical magnetic feature can be seen (see Fig. 1).

Using CONFIT fitting software [8], magnetic part of the spectrum can be decomposed into three components, the hyperfine field of one of which is close to that of pure Fe $\left(B_{h f 0} \approx 32.7 \mathrm{~T}\right)$ and the mean values of two others are lower $\left(B_{h f 1} \approx 27 \mathrm{~T}, B_{h f 2} \approx 24 \mathrm{~T}\right)$ (Table I). Parameter $B_{\text {dist }}$ represents hyperfine field distribution width. Similar fitting model was used by Petrov et al. [5]. The sextet with hyperfine field of one of which is close to that of pure bcc $\alpha$-Fe $(33 \mathrm{~T})$ can be described as iron rich $\alpha$-phase with almost no Cr impurity in bcc lattice. Two other sextets with hyperfine distribution can be assigned to bcc iron with gradually increased $\mathrm{Cr}$ content in the matrix depending on decrease of mean hyperfine field value $\left(B_{h f}\right)$. Relative areas of spectral components can be used for relative quantification of phase composition.

All of the ferritic samples measured by MS spectrometry featured similar components in the iron bearing phases of the analysed steels. Based on $\mathrm{Cr}$ content, relative areas of individual spectral components differ, as well as mean hyperfine field of $\mathrm{Fe}-\mathrm{Cr}$ components, but it seems that chosen fitting model describes all samples well. Chosen fitting model consists of pure bcc $\alpha$-Fe, bcc Fe with Cr substitution and in the case of EP 450 sample also small paramagnetic bcc Cr-rich component.

Positron annihilation lifetime spectroscopy was applied on as-received samples and after gamma irradiation. Positron lifetime measurements were carried out using ${ }^{22} \mathrm{Na}$, with activity about $20 \mu \mathrm{Ci}$, deposited on a kapton foil. The source was sandwiched between two identical samples of steel. A minimum of $1.3 \times 10^{6}$ counts was collected for each of the three runs conducted for individual samples. Positron lifetime data was evaluated using PATFIT-88 software. A detailed description of the resolution function required for spectrum analysis used three Gaussian functions with intensities $80 \%, 10 \%, 10 \%$, and appropriate relative shifts.

The lifetime spectrum is analysed as a sum of exponential decay components, convoluted with the Gaussian functions describing the spectrometer timing resolution using POSITRONFIT. Decay components due to annihilation in $\mathrm{NaCl}(\approx 430 \mathrm{ps})$ and kapton foil $(\approx 382 \mathrm{ps})$ were subtracted in the procedure.

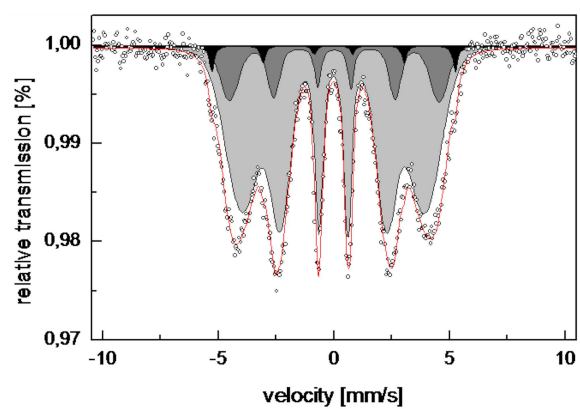

Fig. 1. MS spectrum of sample KOC4-3. Black component represents bcc Fe, dark grey component represents bcc Fe with few Cr substitutions in the lattice, light grey component represents bcc Fe with more $\mathrm{Cr}$ substitutions than in the previous component.

MS spectral parameters of samples

TABLE I

KOC4-3, KOC5-3, KOC6-3, and EP450.

\begin{tabular}{c|c|c|c|c|c}
\hline \hline Comp. & $\begin{array}{c}A_{\text {rel }} \\
{[\%]}\end{array}$ & $\begin{array}{c}\text { IS } \\
{[\mathrm{mm} / \mathrm{s}]}\end{array}$ & $\begin{array}{c}B_{h f} \\
{[\mathrm{~T}]}\end{array}$ & $\begin{array}{c}B_{\text {dist }} \\
{[\mathrm{T}]}\end{array}$ & $\begin{array}{c}\Gamma \\
{[\mathrm{mm} / \mathrm{s}]}\end{array}$ \\
\hline$\alpha$ & 3 & -0.01 & 32.7 & - & 0.25 \\
$\mathrm{Fe}-\mathrm{Cr}$ & 16 & 0.02 & 28.2 & 4.8 & 0.28 \\
${\mathrm{Fe}-C r^{\prime}}^{\prime}$ & 81 & -0.03 & 24.5 & 9.8 & 0.28 \\
\hline \multicolumn{6}{c}{ KOC5-3 } \\
\hline$\alpha$ & 3 & 0.05 & 30.1 & - & 0.25 \\
$\mathrm{Fe}-\mathrm{Cr}$ & 31 & 0.01 & 26.4 & 5.6 & 0.33 \\
$\mathrm{Fe}-\mathrm{Cr}{ }^{\prime}$ & 66 & -0.04 & 24.1 & 10.3 & 0.24 \\
\hline \multicolumn{6}{c}{ KOC6-3 } \\
\hline$\alpha$ & 11 & -0.02 & 32.9 & - & 0.24 \\
Fe-Cr & 37 & -0.01 & 27.7 & 7.5 & 0.30 \\
Fe-Cr' & 52 & -0.03 & 23.5 & 10.3 & 0.29 \\
\hline \multicolumn{6}{c}{ EP450 } \\
\hline$\alpha$ & 5 & 0.00 & 34.2 & - & 0.24 \\
Fe-Cr & 21 & -0.02 & 31.1 & 3.8 & 0.24 \\
Fe-Cr & 73 & -0.02 & 27.4 & 7.6 & 0.25 \\
$\alpha^{\prime}$ & 1 & 0.01 & - & - & 0.48
\end{tabular}

Samples were irradiated at the Washington State University Nuclear Radiation Center using gamma rays generated by a ${ }^{60} \mathrm{Co}$ source. The ${ }^{60} \mathrm{Co}$ irradiator consists of a rod of radioactive Co metal which is stored in a pool of water at a depth of $7.6 \mathrm{~m}$. The ${ }^{60}$ Co source material is adjacent to a $15 \mathrm{~cm}$ diameter aluminum tube which extends from above the surface of the pool down to the ${ }^{60} \mathrm{Co}$ source. The aluminum tube is sealed on the end which is at the bottom of the storage pool and is open to the atmosphere above the surface of the pool water; this allows samples to be placed in a basket to be lowered down into the aluminum tube and positioned adjacent to the ${ }^{60} \mathrm{Co}$ without having to immerse the samples in the storage pool water. The activity of the ${ }^{60} \mathrm{Co}$ source was $6.09 \mathrm{TBq}$ and the irradiation was conducted continuously for $1742 \mathrm{~h}$. The distance from the ${ }^{60} \mathrm{Co}$ to the ODS samples was $9 \mathrm{~cm}$. Sample temperatures were held at $30 \pm 5^{\circ} \mathrm{C}$ during the irradiation.

The results obtained from positron annihilation lifetime spectroscopy was fitted by three exponential 
components where only the first two are interesting in characterization of studied materials $\left(\mathrm{LT}_{1}, L T_{2}\right)$. The average lifetime value was calculated using Eq. (1):

$$
L T_{\text {avg }}=L T_{1} I_{1}+L T_{2} I_{2} .
$$

It is logical to assume that the shorter component $L T_{1}$ arises from annihilations of delocalized positrons. The longer component $L T_{2}$ represents contribution of the positrons trapped at one or more types of defects. The second component $L T_{2}$ for all the samples in as-received state is in the range $197-217 \mathrm{ps}$. This range corresponds to divacancies and suggests that positrons most likely get trapped and annihilate at defects of the same kind in all samples. The concentration of these defects in samples KOC4-3 and KOC5-3 is 55, 57\% respectively; however in KOC6-3 the concentration is about $42 \%$ and I1 is much higher in comparison to materials KOC4-3, KOC5-3. Sample KOC6-3 contains much less defects in base state then the previous. By TEM technique we also asserted that small defects like dislocations are present in the samples.

It is generally believed that the dislocation line represents only a shallow positron trap. The positron diffuses until it finds a vacancy attached to the dislocation, where it afterwards annihilates. From TEM we saw that dislocations get trapped around yttrium particles [9]. Therefore we believe that the positron diffuses to a divacancy (from $L T_{2}$ value) attached to the dislocation which is surrounded by $\mathrm{Y}_{2} \mathrm{O}_{3}$ particles. The reduced positron lifetime $L T_{1}$ moves around $83-100$ ps and is in strong relation with $L T_{2}$ due to the fitting approach. The average lifetime in all samples is around $147 \pm 1$ ps before gamma irradiation [10].

After gamma irradiation we can observe major change in positron lifetime. Both lifetimes increased significantly. Lifetime $L T_{2}$ indicates formation of large clusters of vacancies in KOC4-3, KOC5-3 and vacancy clusters of about 4 vacancies in KOC6-3. The intensities also drastically shifted. After gamma irradiation $I_{2}$ decreased below $30 \%$ in all cases. Intensity $I_{1}$ on the other hand increased above $70 \%$. This indicates that gamma irradiation formed larger defects but with smaller intensity. Divacancies merged into larger defects forming vacancy clusters. Again, sample KOC6-3 seems to have fewer defects and is therefore more immune to gamma irradiation. The main difference in this steel is almost $0.6 \mathrm{wt} \%$ content of hafnium and no zirconium additions. In Ref. [11] they implanted hafnium ions into foils of $9 \mathrm{wt} \% \mathrm{Cr}$ ferritic steels to study the effect of hafnium on the grain boundary precipitation kinetics. It was found that the implantation of hafnium into the steel completely prohibited the formation of the common grain boundary $\mathrm{M}_{23} \mathrm{C}_{6}$ particles. In our case we can assume similar behaviour. Instead of $\mathrm{M}_{23} \mathrm{C}_{6}$ carbides hafnium forms MX type precipitates which increase the concentration of chromium in the matrix and is expected to improve the intergranular corrosion resistance of the material. We do not state that all $\mathrm{M}_{23} \mathrm{C}_{6}$ formation was denied by hafnium, however we believe that hafnium plays a significant role in precipitation kinetics.

\section{Conclusion}

Three ODS steels foreseen for application in advanced nuclear reactors with different chemical content were investigated by transmission electron microscopy, the Mössbauer spectroscopy and positron annihilation spectroscopy and compared to EP450 steel. Samples designated as KOC4-3 and KOC5-3 seem to have very similar properties as to defect type and concentration. Third sample KOC6-3 which is the only sample lacking zirconium and containing hafnium instead shows different behaviour as to initial microstructure and irradiation resistance after gamma irradiation. We attribute this improved resistance to hafnium which to some extent prohibits formation of $\mathrm{M}_{23} \mathrm{C}_{6}$ and forms hafnium carbides that have beneficial effect on the irradiation resistance.

We tied to demonstrate a novel approach how to induce and investigate defects in advanced steels. A high gamma irradiation source and appropriate irradiation time has proven to be sufficient by inducing major defects to the microstructure. Gamma irradiation does not interfere with positron measurements or TEM by undesirable side effects like in the case of neutron irradiation. In our future work we will also compare neutron irradiation and gamma irradiation on these samples. Nevertheless we believe that gamma irradiation of steels could be a helpful defect inducing method lacking the disadvantages of classical neutron irradiation.

\section{Acknowledgments}

Authors acknowledge grants VEGA 1/0214/13, $1 / 0339 / 16$ and 001STU-2/2014. The help of Dr. Maria Domankova in TEM analyses is highly appreciated.

\section{References}

[1] R. Kasada, S.G. Lee, J. Isselin, J.H. Lee, T. Omura, A. Kimura, T. Okuda, M. Inoue, S. Ukai, S. Ohnuki, T. Fujisawa, F. Abe, J. Nucl. Mater. 417, 180 (2011).

[2] S.M. Dubiel, K. Krop, J. Phys. (France) 12, C6-459 (1974).

[3] S.M. Dubiel, J. Cieślak, Crit. Rev. Solid State Mater. Sci. 36, 191 (2011).

[4] D. Chandra, L.H. Schwartz, Metall. Trans. 2, 511 (1971).

[5] Yu.I. Petrov, E.A. Shafranovsky, Yu.F. Krupyanskii, S.V. Essine, J. Appl. Phys. 91, 352 (2002).

[6] R. Idczak, R. Konieczny, J. Chojcan, J. Phys. Chem. Solids 73, 1095 (2012).

[7] P.A. Flinn, S.L. Ruby, Phys. Rev. 124, 34 (1961).

[8] T. Žák, Y. Jirásková, Surf. Interface Anal. 38, 710 (2006).

[9] I. Bartošová, M. Dománková, F.A. Selim, V. Slugeň, J. Phys. Conf. Series 618, 012014 (2015).

[10] I. Bartošová, Ph.D. Thesis, STU Bratislava, Slovakia 2016.

[11] Y.F. Yin, R.G. Faulkner, Mater. Sci. Tech. 21, 1239 (2005). 